InVisible Culture Journal

\title{
Playful (Counter)Publics: \\ Game Mods as Rhetorical \\ Forms of Active and \\ Subversive Player \\ Participation
}

Nicole Kurashige ${ }^{1}$

${ }^{1}$ University of Hawaii

Published on: Apr 18, 2019

DOI: $10.47761 / 494 \mathrm{a} 02 \mathrm{f} 6.76 \mathrm{a} 37257$

License: Creative Commons Attribution 4.0 International License (CC-BY 4.0). 


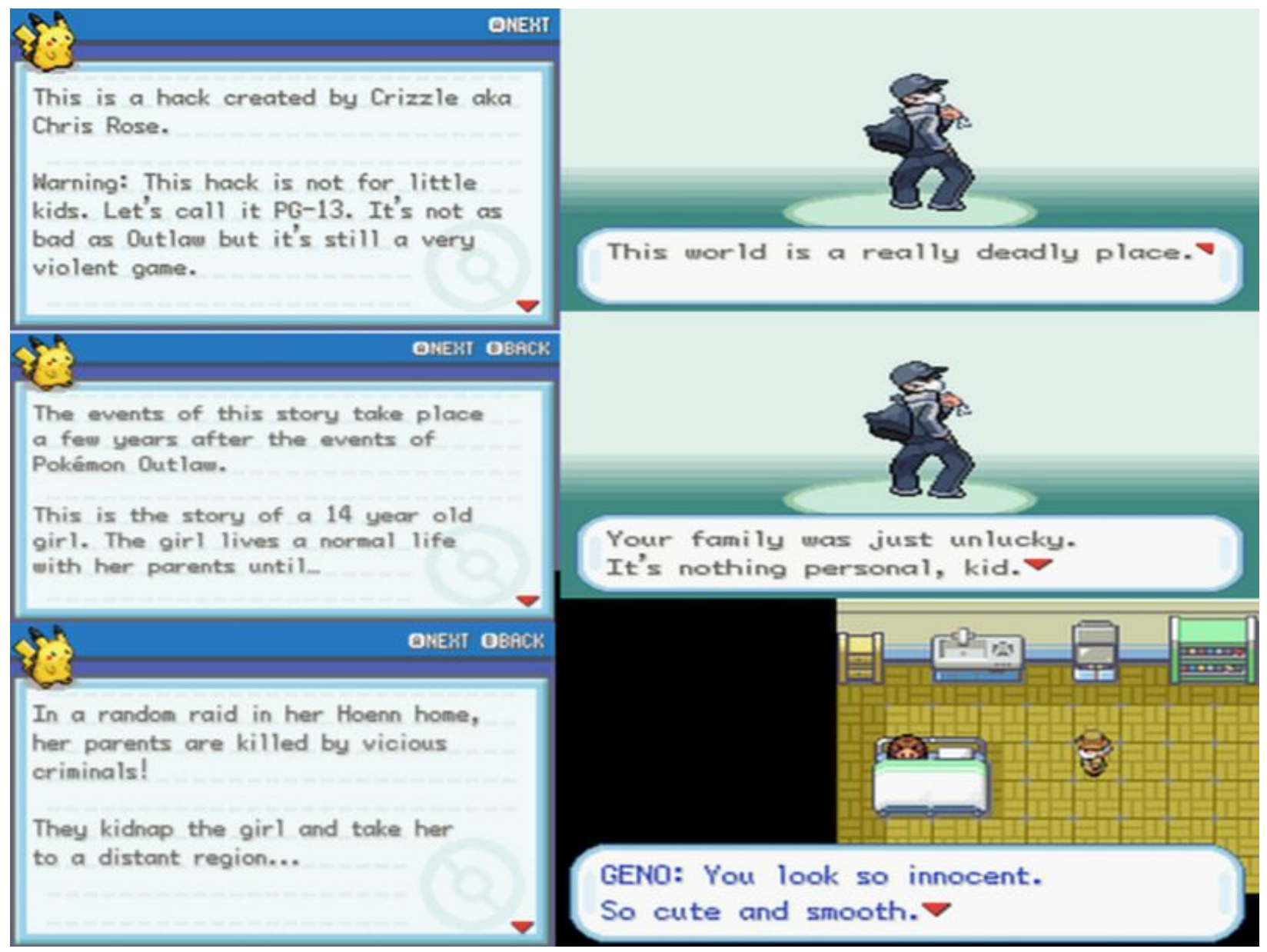

Pokémon Korosu In-Game Screenshots

\section{Introduction}

Though most digital humanities scholars readily agree that game developers need to offer more progressive functions and options to enhance player agency, such recommendations for further research or action often ignore how players are already able to enact their agency in spaces beyond the game itself. Online gaming forums serve as hotbeds of active player participation and (counter)public discourse. $\underline{1}$ Players seeking to expand their agency within games can do so via modifications (referred to hereafter as "mods"). $\underline{2}$ Mods, which are collaboratively developed in such online forums by players for players, are digital compositions that can alter the code of a game in various ways, thereby opening up more possibility spaces for players without having to wait for developer intervention. Players, thus, challenge, resist, and subvert the procedural rhetoric encoded in a game by exhibiting their agency through the 
creation, distribution, and use of these mods. Mods and their related modding communities have been around for decades, but, surprisingly, little to no research has been done to examine their rhetorical significance. This article seeks to draw attention to the intellectual and practical benefits this rich but untapped area might yield for digital composition specialists and researchers.

The Pokémon video game series (1996-2017) developed by GameFreak and published by Nintendo is part of a larger multimedia franchise that includes television shows, movies, and merchandise. In its twenty-two years of existence, the general premise of all official Pokémon games remains the same. Set in a fictional universe where creatures called Pokémon coexist with humans, the player takes on the role of a child who sets off on a quest to be the best Pokémon trainer in the world. $\underline{3}$ To win the game, players have only three main goals they must achieve: 1) to form and train a team of Pokémon to compete against other teams owned by other trainers in order to eventually "battle" their way to the top and become the regional champion, 2) to complete the regional Pokédex by collecting all the available Pokémon species found in a particular fictional region, and 3) to complete the national Pokédex by collecting all the available Pokémon from different regions via trading Pokémon with other players or transferring Pokémon from other games. With the recent addition of Pokémon Sun and Moon (2016-2017), there are now over 802 Pokémon to capture, train, and trade. From a technological standpoint, the longstanding popularity and success of the Pokémon series is a testament to its solid coding, which has been tweaked and perfected with each game. Having a familiar code to return to has normalized players' expectations of the series and, thus, further solidifies their willing participation in their self-interpellation in what Nintendo markets as wholesome ideologies.

The following article examines how Pokémon Uranium (2016) and Pokémon Korosu (2016), two unofficial spin-offs of Nintendo's Pokémon (1996-2017)series, are examples of how fan-made mods can be used to subvert the procedural rhetoric of the original games and create (counter)public discursive communities via online forums where players converge and collaborate. Procedural rhetoric refers to how the mechanics of a game result in the perpetuation of certain ideologies via player interaction. $\underline{4}$ Values embedded within video games can range from promoting wholesome behaviors such as neighborliness to encouraging complex intellectual interrogations of capitalistic economic structures. As recognizable patterns of what is allowed and disallowed soon emerge, players begin to form their own strategies of the best ways to respond to the dynamics coded within the games via procedural rhetoric. However, as Kevin Brock and Dawn Shepherd observe "[p]rocedural systems are not 
neutral, inert tools [...] they are complex and active rhetorical agents who make arguments to and through us via the activities they assist us with, and hinder us from, completing." $\underline{5}$ Players who do not fall within the ideological majority might find themselves limited by the procedural structure of the game. It is in these instances that mods can offer players the opportunity to not only experience a game outside of its original coded intent, but also provide the opportunity to be an integral component of a shared community of fellow players (e.g. gaming forum), thus creating a kind of (counter)public discursive space.

Public discursive communities often circulate values and ideologies that are shared by the majority, thus marginalizing or silencing those who do not readily conform to such standards. Counterpublics, as Nancy Fraser asserts, are formed in response to an exclusion from the dominant publics, and can serve as discursive arenas in which "members of a subordinated social group invent and circulate counter discourses to formulate oppositional interpretations of their identities, interests, and needs."트 Modding, by Fraser's standards, is a countercultural action that has the potential to create a counterpublic discursive community through the creation and distribution of code that resists or departs from commercialized narrative structures in games. Understanding the motivation and use of mods as an active form of player resistance to video game developers and their prepackaged narratives is, therefore, essential in unpacking the relationship between modding and ongoing studies about the ethics of cultural remixing and the circulation of non-profit digital compositions. Pokémon Uranium and Pokémon Korosu subvert the ideological beliefs presented in the official, licensed Pokémon titles by challenging the notion that narratives of childhood experience must, by default, be happy and free of all forms of emotional conflict and trauma. The procedural rhetoric of Nintendo's commercialized Pokémon games often deemphasizes some of the harsher realities of life to further a wholesome worldview. These beliefs are often encoded into the game as distinct rules, which limit the player's ability to deviate from what Nintendo deems as the norm. While there is value in Nintendo's promotion of an exciting childhood adventure, many of their games serve as escapist fantasies that omit the darker elements of ongoing sociocultural, economic, and political strife.

\section{Subversive Code: A Closer Look at Pokémon Uranium and Pokémon Korosu}

Fans of the Pokémon gameseries are enamored with its picturesque version of innocence that promotes children's agency above all else. Nostalgia for the past, even 
if it is a past riddled with questionable situations, is a powerful emotion that drives players to pick up the latest game that Nintendo has to offer in order to re-live such fantasies all over again. As Keza Macdonald explains in "Why I'm a Full-Grown Adult Who Still Loves Pokémon," "Pokémon is a classic story of child empowerment, set in a world that's largely free of adults, in which kids go out into the world and make their own way." 7 Macdonald points to the ability for players to express themselves via the creation of their Pokémon team, of which there are 802 "digital pets" to choose from along with an avatar customization system included in recent iterations of the game. $\underline{8}$ Her assertion, however, that "Pokémon is an exploration-rich game that lets you do things how you want" is incomplete and imperfect when considering the limiting factors of procedural rhetoric. .9 Jason Schreier's "Why I Can't Get Into Pokémon" (2013) points to such limits within the game's narrative and coding structure as reasons why the Pokémon games fail to attract and maintain his attention:

In Pokémon, your characters are cyphers, because they're animals. They're Pokémon. You can stamp part of yourself on each one of them, training and breeding and customizing their abilities, but you can never make them talk, or tell them to interact, or have them do much of anything other than fight with one another. When I play an RPG, I don't want cyphers; I want people. I want to go on an adventure with people who have motivations and flaws and conflicts and relationships, and I want to see how they evolve-both narratively and mechanically -as they overcome those obstacles [...] In Pokémon, your journey is to go take down other Pokémon trainers, and if you don't immediately develop a desire to collect critters and battle them against one another, there's not a whole lot to drive you forward. $\underline{10}$

The existence of Pokémon mods, thus, is a direct response to players' desires to resist, challenge, or subvert the procedural rhetoric of mainstream Pokémon games to explore deeper player possibilities. Pokémon Uranium (2016) and Pokémon Korosu (2016) are examples of mods thatattempt to add depth to the "one-note" world in which "no one talks about anything except Pokémon" that Schreier and other players have, perhaps, grown too accustomed to over the years. $\underline{11}$ 


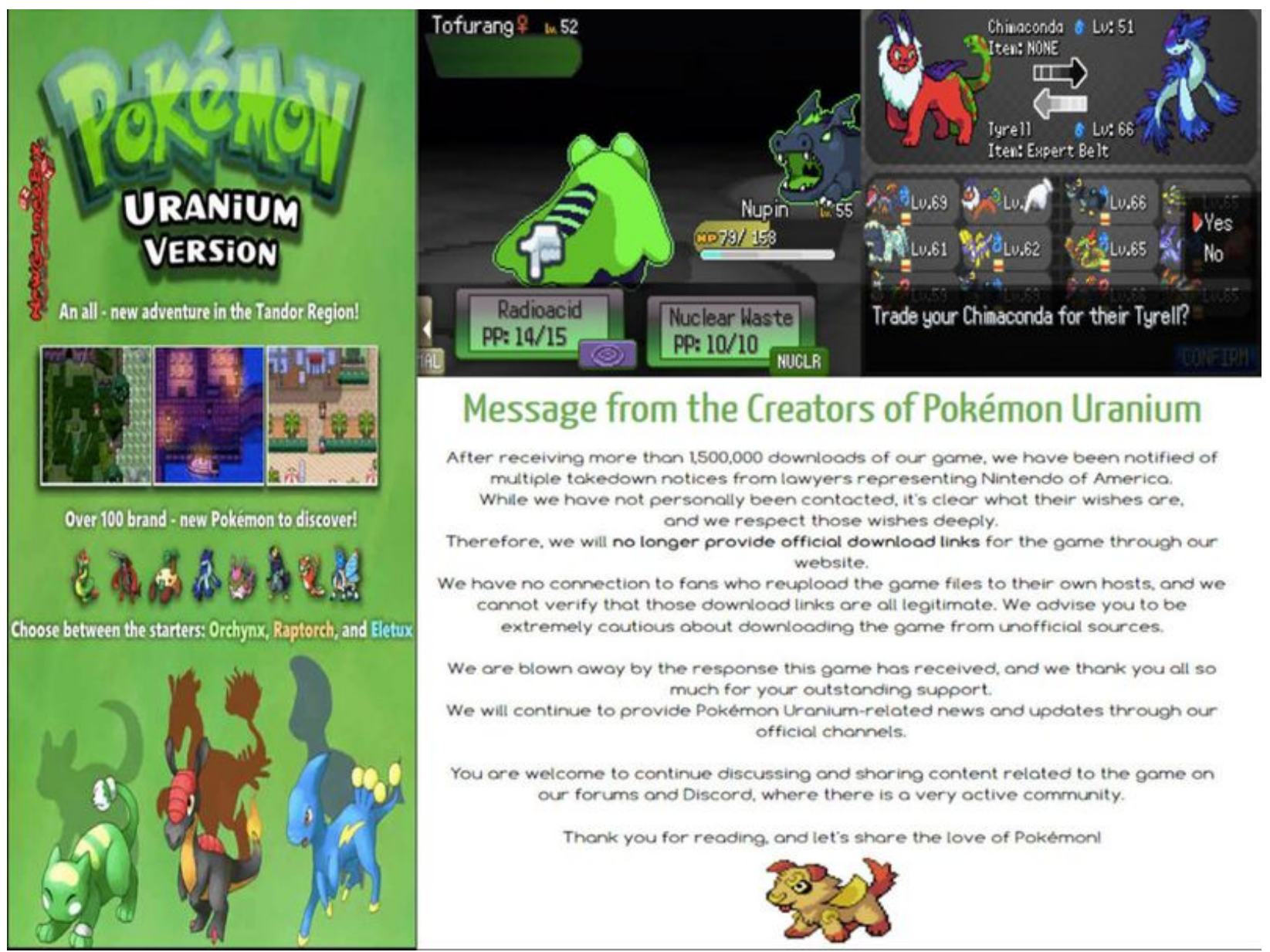

Figure 1: Pokémon Uranium In-Game Screenshots and 2016 DMCA Takedown Notice

Pokémon Uranium is a non-profit, fan-made spin-off title of the popular Pokémon series by Nintendo (figure 1). According to Nick Monroe's Gameranx article "All About Pokémon Uranium" (2016), this game is the culmination of over nine years of work by the duo $\sim \mathrm{JV} \sim$ and Involuntary Twitch. $\underline{12}$ By heavily re-writing the code present in previous Pokémon titles through the RPG Maker XP engine, the modders sought to provide a new gameplay experience for fans of the original series with better mechanics and a deeper story. The resulting modded game added over 150 new fanmade species of Pokémon along with a new region to explore, as well as brought up issues related to nuclear armament. On August 6, 2016, the first "official" download for Pokémon Uranium was released via the Internet and was met with resounding praise from players worldwide. Within its first week of release, the game was downloaded over one and a half million times. $\underline{13}$ The rapid and massive success of Pokémon Uranium soon drew unwanted attention from Nintendo executives in both America and Japan, thereby resulting in multiple DMCA takedown notice letters from 
corporate lawyers. By August 13, 2016, JV and Involuntary Twitch announced via the "official" Pokémon Uranium Twitter account that they "will no longer provide official download links for the game" due to the threat of legal action against them, but encouraged others in the gaming and modding communities to continue discussing and sharing their work. $\underline{14}$

In Pokémon Uranium, the avatar the player embodies is the child of Pokémon ranger Kellyn and nuclear technician Lucille. $\underline{15}$ Ten years before the events of the game, Lucille is killed in an explosion at a nuclear power plant, which leaves Kellyn in a state of disarray. Unable to handle his depression, Kellyn throws himself into his work and leaves his child, the avatar, in the care of an abusive relative. The player, subsequently, explores the physical and emotional impacts of the nuclear explosion and a father with unresolved grief and survivor's guilt. While the original battling and collecting system remains part of the mod, Pokémon Uranium's ultimate end goal is less about making it to the Pokémon League and more about mending the broken relationship between parent and child. Pokémon Uranium, in this regard, responds to the absent father trope found across all licensed Pokémon games. The official games follow a traditionally Japanese cultural frame of reference where fathers are excused from participating in their children's lives because they are actively working to gain financial stability for their families. Nintendo, thus, emphasizes the importance of family values where everyone follows a strict role: mothers are homemakers, fathers are breadwinners, and children are carefree and innocent. This situation is clearly not the case in Pokémon Uranium where the child is forced to nurture their parent while also struggling to survive on their own. In Pokémon Uranium, the extent to which the player is able to re-establish a familial connection with Kellyn is dependent upon their choices and actions within the game. The inclusion of branching quest lines allows players the possibility of understanding, but not necessarily accepting, the reasons why some families fall apart, thereby resisting Nintendo's predisposed urge to ignore such topics. 


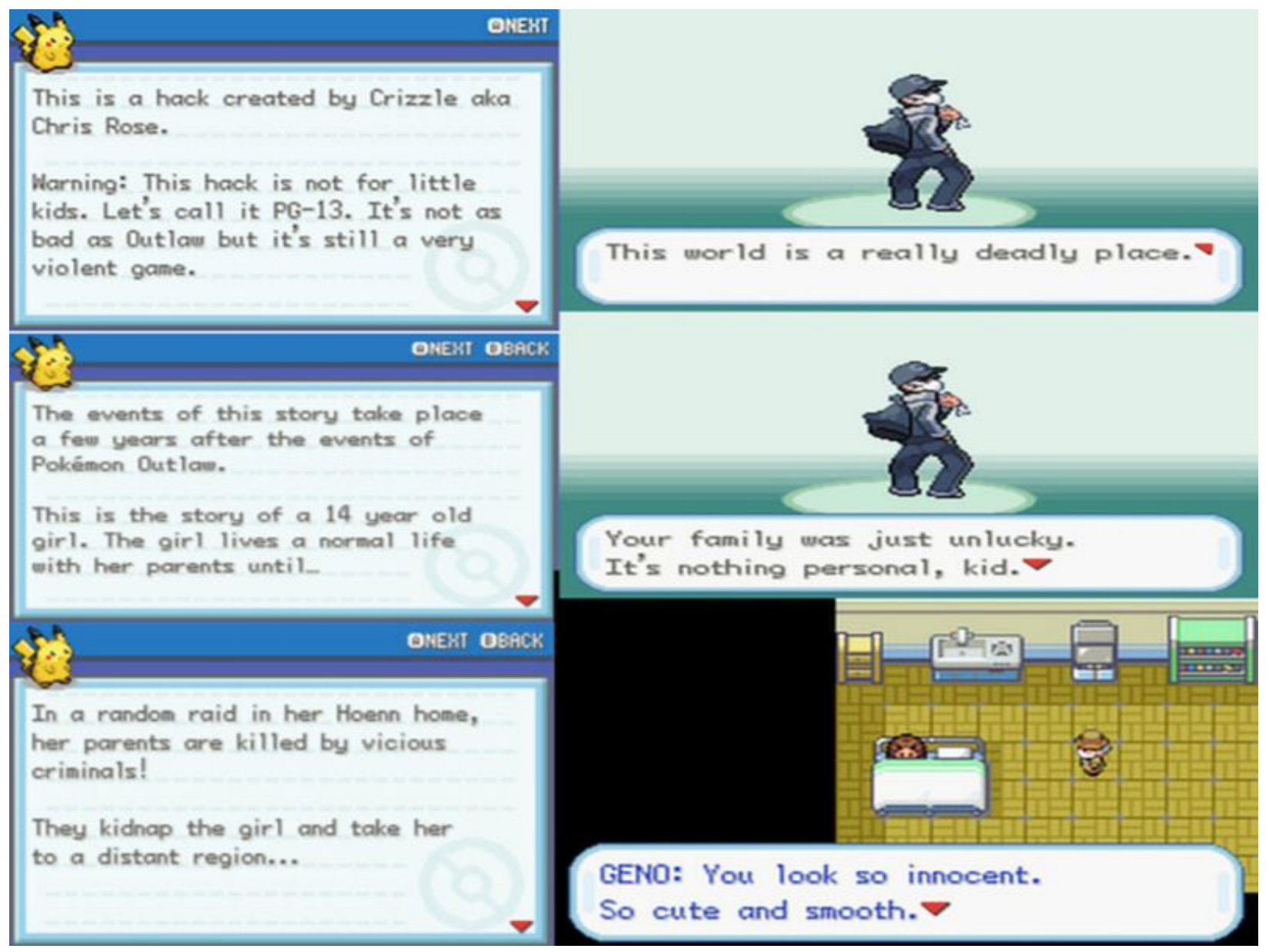

Figure 2: Pokémon Korosu In-Game Screenshots

The potential that mods have in creating Pokémon games that are simultaneously familiar and unfamiliar can also be seen in Pokémon Korosu (2016), another non-profit, fan-made spin-off. Although Pokémon Korosu (Fig. 2) was released a month before Pokémon Uranium, it was met with less explosive reception due to its limited niche audience. Pokémon Korosu, developed by Crizzle (Chris Rose) is an ultra-violent reimagining of the Pokémon series. The term "korosu" can be roughly translated from Japanese to English as "murder" or "kill." 16 As such, the focus of this particular game is to see how far players will go to ensure that the main protagonist achieves her quest for revenge. Drawing upon the Kill Bill film series for inspiration, Crizzle sought to create a Pokémon game where players embody the identity of a young girl who witnesses the slaughter of her entire family and is, thus, kidnapped and sold into a human trafficking ring. At different points in the story, players are allowed to make moral and ethical decisions that impact the girl's psyche. 17 Though Pokémon Korosu has received generally favorable reviews from players looking for a darker and grittier gaming experience, its complete departure from the original series' innocent tone 
resulted in the game's predominantly underground success. This lack of mass attention, however, seems to work in the game's favor, as Crizzle has made no announcements about receiving DMCA takedown notices or Cease and Desist letters from Nintendo. $\underline{18}$

Pokémon Korosu, which is massively different from the Nintendo's licensed Pokémon games and other fan-made games such as Pokémon Uranium, speaks to the extent that mods have in not only resisting and subverting ideological beliefs, but also in establishing a platform for (counter)public discourse to occur. According to Crizzle's developer notes, his Pokémon game hypothesizes how horribly situations could go in the Pokémon universe if the narrative wasn't distilled in the controlled and wholesome way that Nintendo usually presents the series. $\frac{19}{19}$ The darker atmosphere of the game draws attention to issues regarding the loss of childhood innocence and the horrors of sexual violence and abuse. As part of her quest for revenge, the main heroine of Pokémon Korosu has the option of killing or maiming her human captors and opponents by setting her Pokémon on them. $\frac{20}{}$ Choosing to partake in this eye-for-aneye approach to justice is the main staple of the game and, thus, serves as an invitation for players to question whether such force is warranted, even with the knowledge that the main heroine has suffered at the hands of other humans. Unlike Pokémon Uranium, where players can change the play settings, Pokémon Korosu automatically codes the Nuzlocke Challenge as a "fixed" feature to add weight to the player's

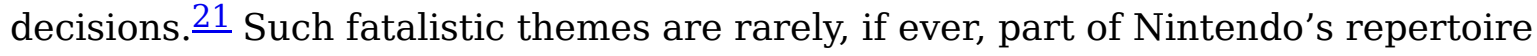
because of their strict adherence to developing games that the whole family can enjoy: "At Nintendo, our mission is to put smiles on the faces of anyone who experiences our brands." $\underline{22}$ Though official Pokémon titles include a fair share of in-game violence in the form of Pokémon battles, the seriousness of these situations is downplayed by the game's recovery system, thereby removing the threat of consequences for one's actions. $\underline{23}$

Pokémon Uranium and Pokémon Korosu are some of the most recent instances of how players recognize the procedural rhetoric embedded in games and, subsequently, find alternate ways to resist or subvert such imposed values, rules, and restrictions. Unlike official Pokémon games, modded games like Pokémon Uranium and Pokémon Korosu do not offer players such an easy escape into a childhood fantasy where the real world does not matter. The decisions made in these mods cannot be changed unless the player decides to wipe their data and start all over again, forcing players to remain conscious of their actions. Mods, in this regard, can be used to create what Bryan 
Bergeron (2006) calls "serious games": “[m]ost mods are intended to create new, improved, entertainment games...[but] the mod process can also be used to create serious games." $\underline{24}$ The fear of impending nuclear crisis, the dark realities of human trafficking, and the tragedy of broken or abusive households and families are some issues that players, both adults and children, might grapple with or witness in their communities outside the game. Even if players are unaffected by such heavy issues, the themes in these modded fan-games can function as stand-in contextual frames for a whole host of general feelings such as fear, anxiety, and isolation. Pokémon Korosu's narrative structure and gameplay mechanics peel back the layers of decorum that Nintendo encodes into their official games by making such ideological conundrums its primary focus. The coding of Pokémon Uranium also counters Nintendo's claim that an ideal childhood is one that is completely free of adult supervision. As a general rule, the avatars that players control in Pokémon games are between the ages of ten and thirteen in order to maintain the idea that children have the power to "run the world." $\underline{25}$ But child agency does not negate the need for adult guidance or intervention, especially considering that the children in commercial Pokémon games are burdened with journeys that possess a great deal of danger.

Mods are, therefore, essential in pushing back against the commercial production of games coded to ignore the role dominant ideologies have in supporting the obfuscation of other positions and realities. The creation of Pokémon Uranium and Pokémon Korosu marks an interesting turn in how players actively recognize and respond to the limitations and biases coded within a game and develop ways to oppose such systems. While the Pokémon collection and battling system is the same as the licensed version, the difference in the recovery mechanics and narrative structures of these modded games highlights how much players can deviate from Nintendo's predetermined script. Furthermore, the growing presence of sophisticated mods such as Pokémon Uranium illustrate how modders can experiment with other possibility spaces that do not to replicate or adhere to the ideological hallmarks that Nintendo deems necessary in creating a "good" Pokémon game. Though the Pokémon Uranium modders ultimately chose to cease their development and shut down their official website and servers, the mod file for the game can still be found and downloaded on other non-affiliated fan sites and forums. As of 2018, fans of Pokémon Uranium are continuing the work of $\sim \mathrm{JV} \sim$ and Involuntary Twitch by modifying existing code structures and adding new content to the base mod to further deepen and enhance players' experiences. Similarly, Pokémon Korosu enjoys a measure of self-contained success through its active community discussion board. Mods, from this standpoint, are part of an open-source 
community where everyday players can become cultural producers in their own right, which can provide scholars with a valuable point-of-reference to how such digital compositions can be leveraged for further research. $\underline{26}$

\section{The Significance of Fan-Made Modifications in Expanding Player and Researcher Possibility Spaces}

The differences between the coding of modded fan-games like Pokémon Uranium and Pokémon Korosu and licensed Pokémon games demonstrates the extent to which players recognize and respond to the limitations and shortcomings of Nintendo's procedural rhetoric. Mods are full of rhetorical and pedagogical potential due to their power to completely alter gaming experiences in various ways. Modding has been described as a kind of do-it-yourself approach to technology personalization that is often used to tailor a game to an individual's personal and stylistic tastes (e.g. character design, character gender, race, sexuality, character romance options, etc.), but this personalization goes well beyond changing the aesthetic or interface settings of a game. Mods, as Hector Postigo claims, "can be resistive" even when they are sometimes "purely an outcome of hobbyist intentions." $\underline{27}$ With the majority of Pokémon players being between the ages of nineteen and twenty-four, it is clear that this particular gaming community is in the process of challenging Nintendo's optimistic ideological beliefs by creating alternate options that, perhaps, reflect a worldview carried by an audience who has long since outgrown the artificial happiness of Pokémon in favor of the more mature perspectives found in Pokémon Uranium and Pokémon Korosu. $\underline{28}$

Because commercial games offer players minimal room to challenge the underlying issues encoded and enforced by a game's procedural rhetoric, players are faced with the inevitable choice of seeking possibility spaces elsewhere. The increasing availability and accessibility to digital tools and media has made it so that anyoneeven a player seeking to expand their possibility spaces within a game-can be a cultural producer. In Available Means of Persuasion: Mapping a Theory and Pedagogy of Multimodal Public Rhetoric, David M. Sheridan, Jim Ridolfo, and Anthony J. Michel discuss how an increasingly digitized society has made resistance the work and responsibility of not just a few highly trained individuals, but all people. $\underline{29}$ More specifically, Sheridan et. al draw attention to how the creation of the "First Things First" ("FTF") Manifesto highlights a key problem educators, researchers, and, most importantly of all, rhetors face $\underline{30}$ : “[w]e are immersed in discourses produced by technically proficient and highly creative culture workers whose talents serve media 
institutions that are ultimately interested in profit."쑤 For-profit developers, such as Nintendo, control the procedural rhetoric in their games as well as the marketing and distribution of said games. Thus, instead of waiting for developers to create codes that challenge, resist, or subvert dominant ideologies, players now possess the ability to take control of games for themselves through mods. Modding requires less effort than building an entire game from scratch and also allows players, who are usually fans of the franchise, to tailor their favorite games to fit their personal fantasies. The more demand there is for a particular kind of mod, the more likely someone in the modding community will respond by creating and distributing it for free via online fan forums. The action of modding is, therefore, not only spurred by individual impetus, but also can be a direct result of community engagement.

When ordinary people appropriate the rhetorical tools of graphic artists, photographers, illustrators, and game designers, the power of cultural production is placed in their hands. Thus, digital, new media, and multimodal rhetoric and composition are an alternative way to actively resist and, sometimes, subvert, the larger institutionalized structures that continually threaten to silence, control, obscure, and/or exploit (counter)public thought-which, in and of itself, can become something extraordinary. As such, fan-made mods are propagated in separate spaces from the game itself. Here, fan-made mods of commercial code can provide the tools needed to bypass the closed, pre-determined digital narrative structure in the original or base game and, thereby, allow players to exert their agency in resistive and subversive ways. Modders, people who create modifications, in this regard, become part of an emergent rhetorical process in which they take on the role of "rhetors-aspoints-of-articulation" to address the desires and expectations of a larger, digital (counter)public. $\underline{32}$ Websites such as The Poké Community and PokEdit as well as subthreads housed in preexisting forum websites (e.g. "Play and Create Pokémon ROM Hacks Reddit") all serve as communication hubs for modders and non-modders alike, thus building inclusion within and across online spaces, and increasing the likelihood of alternate or counter narratives to develop and spread. Using community message boards, modders are able to announce their plans to release new content, dispense and receive feedback for troubleshooting errors, and post downloadable links to completed modding projects.

Although Bogost describes video game play as a "community of practice" where "people who play video games develop values, strategies, and approaches to the practice of play itself," he discounts the impact that such communities "outside the 
game" have on procedural rhetoric. $\frac{33}{3}$ But games do not exist in a vacuum, and they are consumed and critiqued by a wide audience. It is the player's interaction with the game that, in turn, shapes the mods that are created and distributed in a particular community. Outside communities, especially those consisting of modders, play a significant role in the resistance and subversion of procedural rhetoric. Modding, therefore, is an extension of video game play because of how modders play with a game's code to change its structure either partially or completely. According to Nathaniel Poor's “Computer Game Modders' Motivations and Sense of Community” (2014), “[m]odding is more than adjusting the preferences or game settings, it is making changes that cannot be made through the game as it is." $\underline{34}$ Poor's comprehensive study of modder motivations reveals that it is the collaborative aspect of the modding process that draws many individuals to their work, as 107 of 111 (96.4\%) survey respondents reported that a sense of "community" was integral to their modding activities. $\underline{35}$ There is no distinction or disconnect between the games modders play and the outside forum communities of which they are a part. Being a player is integral to one's membership within a related forum, as it is difficult to enter such a video game-centric Burkean Parlor without having firsthand knowledge or experience of a game itself. Postigo (2010), whose theories inform much of Poor's analysis, further expands upon the notion of modding as an essential part of active player participation. Postigo's research asserts, "modding is an important characteristic of participatory culture in video games." $\underline{36}$ Modding can, thus, be regarded as an essential discursive act that bridges the mainstream gaming industry and other marginalized or underrepresented player groups/communities.

Recognizing mods as responsive or reactive forms of digital rhetoric and composition requires a closer examination of mods that respond to the need for gameplay experiences that deviate from the rules imposed on players by commercial games (e.g. Pokémon Uranium and Pokémon Korosu). Digital rhetoric and composition is key to participatory culture and poetic world building by allowing people from a variety of sociocultural, political, and economic positions and identities to create, disseminate, and exchange ideas and counter-ideologies. Kairos or timeliness, as mentioned repeatedly by Sheridan et. al, is one of the most important factors when it comes to the rhetorical effectiveness and (counter)public pervasiveness of "new" forms of communication such as video games and mods. $\frac{37}{30 v i n g}$ in and out of the (counter)public sphere is not as easy as entering and re-entering a building, and sometimes the "right time" to act never occurs. Developers may have already released a downloadable update that addresses player critiques for improving a licensed game 
series, thereby downplaying or negating the need for certain kinds of mods.

Furthermore, modders may have an idea for a mod, but those initial concepts may not immediately fit the needs of the gaming community of which they are a part. While many factors can be beyond a modder's control, kairos can still be manufactured as a means of making room for opportunity to occur. Mods are, therefore, digital compositions that emphasize players' awareness of the available options that rest at their fingertips and can be used to create a specific kairotic movement that, in turn, stimulates more collaborative discussion and community engagement.

It might be easy to brush off the impact of video games and default to the notion that play is for the sake of play itself, but it is crucial to recognize and accept that the procedural rhetorics described by Bogost will never be neutral. $\underline{38}$ The rhetoric encoded in games attempts to make and uphold claims about what the best course of action should be and how players should go about achieving their goals to fit a particular ideological mold. There will, thus, always be something at stake in regards to how players-many of whom are college-aged students-critically engage with their consumption of commercialized games and other new media objects/texts. To believe that the act of play cannot materialize in real physical or psychic harm is to seriously misjudge the capabilities that it possesses as a means of interpellation. Just as critical reading is a core skill students must build throughout their years in college-level writing courses, so too must the skill of critical play be emphasized. If composition specialists and researchers want their students to begin critically examining how digital compositions such as games are designed around dominant ideological norms that shape the modes of production, power, and authority, they must be willing to also engage with the rich discursive communities that online forums have to offer. Entering into these discussions, thus, requires that academics to learn how to play critically themselves, as their participation in games is necessary to the development of this field of research.

By literally recoding systems, either in its entirety or select parts, modders are able to hack open new pathways for other players to explore. Walter Scacchi, in "Computer Game Mods, Modders, Modding and the Mod Scene," also notes "modders are players of the games they construct...[t]here is no systematic distinction between developers and users in these [modding] communities." $\underline{39}$ Modders and non-modders are, thus, both responsible for maintaining the rhetorical velocity of mods. $\frac{40}{\text { Replacing the }}$ dominant ideologies that code a game's structure with fan-made content and then distributing said content for free to an entire community of players is an act of 
resistance and subversion against both the base game's embedded procedural rhetoric and the larger corporate entities that dictate what kinds of ideologies should or shouldn't be represented in their products. Who and what gets valued by games and how this ideological representation takes shape via a game's code is integral to interrogating issues regarding the circulation and perpetuation of harmful stereotypes and/or problematic discourse. It is not to say that such studies are meant to extract the entertainment value that games have to offer players. People can still appreciate and enjoy problematic material, but it is important to recognize and articulate why such problems exist and identify ways to challenge them. As Jens Seiffert and Howard Nothhaft claim in "The Missing Media: The Procedural Rhetoric of Computer Games," games are powerful persuasive devices that require more scholarly attention: "the failure of researchers and scholars to devote adequate attention to computer games is a missed chance for communications research." $\underline{41}$ Though Seiffert and Nothhaft's argument focuses on the use of games as tools for public relations research, I contend that their basic call to action can also be extended to the research of mods for use in composition courses.

More work must to be done to fully unpack the benefits that approaching games from these rhetorical, compositional, and pedagogical standpoints have to offer academics. Joshua Daniel-Wariya's “A Language of Play: New Media Possibility Spaces” suggests that "play-like words and images-is a resource used by people to express attitudes, to share ideas, and to persuade others. Such play is at stake at all levels of composing, including invention, production, consumption, distribution, and access." $\underline{22}$ Critical play requires engagement with serious games. Bergeron defines "serious games" as games that "contain a challenging goal, are engaging, and imparts the user with a skill, knowledge, or attitude that can be applied to the real world." $\underline{43}$ This definition can also extend to the process of modding itself. Mods can be just as "serious" as commercial games and can also be leveraged as a process-based learning project in which participants gain hands-on experience with the key concepts needed in creating collaborative digital compositions for both academic and non-academic communities. Critical play and the concept of serious games are crucial in thinking about how mods can be mobilized to better understand how such digital compositions intersect ongoing issues concerning media ethics, collaborative authorship and ownership, and remix culture. Modding, as Poor asserts, "involves many activities that are both important to human beings and are of interest to researchers: collaboration, community, play, digital skills, making, and cultural remixing." $\underline{44}$ Composition scholars and specialists must, therefore, engage with games through their own critical play and involvement in 
online gaming and modding forums. Doing so might yield more fruitful discussions on the importance of civic engagement and responsibility in a digital world. It is my hope that my remarks in this article illustrate the value of mods as digital compositions and highlight the ways modding communities can expand scholarly insight on the rhetorical productivity that occurs in such possibility spaces.

\section{Footnotes}

1. The parentheses in the term (counter)public are used to connote fluidity in its definition when used in reference to mods. Specifically, mods can be used-to various extents-to either reify accepted beliefs circulating within a public sphere or resist such beliefs in an attempt to be countercultural. $\triangleq$

2. I use Nathaniel Poor's theoretical framework to define modifications or "mods" as the process of changing a game's "base code" through the use of computer programming software and tools that are not part of the original game. As Poor notes, "modding is...done by players and fans of the game" and usually operates as an unlicensed, unsupported form of game design. Nathaniel Poor, "Computer game modders' motivations and sense of community: A mixed-methods approach," New Media \& Society 16, no. 8 (2014). $ヒ$

3. “Pokemon," Encyclopaedia Britannica Online, https://www.britannica.com/topic/Pokemon-electronic-game.

4. Ian Bogost, Persuasive Games: The Expressive Power of Video Games (Cambridge, MA: MIT Press, 2007).

5. Bogost, Persuasive Games, 26. $\leftrightarrows$

6. Nancy Fraser, "Rethinking the Public Sphere: A Contribution to the Critique of Actually Existing Democracy," Social Text 25/26 (1990).

7. Keza Macdonald, "Why I'm a Full-Grown Adult Who Still Loves Pokémon," VICE, September 4, 2014, https://www.vice.com/en us/article/7b7dkz/still-love-pokemon$\underline{234} . \underline{-}$

8. Macdonald, "Why I'm a Full-Grown Adult Who Still Loves Pokémon." 9. Macdonald, "Why I'm a Full-Grown Adult Who Still Loves Pokémon." 
10. Jason Schreier, “Why I Can't Get Into Pokemon,” Kotaku, October 18, 2013, https://kotaku.com/why-i-cant-get-into-pokemon-1447775268.

11. Schreier, "Why I Can't Get Into Pokemon."

12. Nick Monroe, "All About Pokémon Uranium: What Happens When Nuclear Energy is Introduced in the Pokémon World?" Gameranx, August 15, 2016), http://gameranx.com/updates/id/69498/article/all-about-pokemon-uranium/.

13. Monroe, "All About Pokémon Uranium."

14. Monroe, "All About Pokémon Uranium."

15. Monroe, "All About Pokémon Uranium."

16. Crizzle, "FireRed Hack: Pokémon Korosu Update," Poké Community

Forums, 2016, https://www.pokecommunity.com/showthread.php?t=372483.

17. Crizzle, "FireRed Hack." $\leftrightarrows$

18. Crizzle, "FireRed Hack."

19. Crizzle, "FireRed Hack."

20. Crizzle, "FireRed Hack." $\doteq$

21. The Nuzlocke Challenge is a set of self-imposed rules intended to create a higher level of difficulty while playing Pokémon games. The basic rules of the challenge are as follows: 1 ) if a Pokémon falls in battle it is considered "dead" and must be released from the player's team, 2) the player may only catch the first Pokémon encountered in each area and none else, 3) all Pokémon must be given a nickname for the sake of establishing stronger emotional bonds between the team and the player, 4) players must only use Pokémon they have captured themselves, 5) if an entire team of Pokémon should fall in battle, it is considered as a "game over," regardless of how may "extra" Pokémon the player has in their storage. The rules are not an in-game function, but some modders have designed codes that allow players the ability to turn the Nuzlocke challenge off or on (the impact of playing a game coded to adhere to these rules will be discussed later in this article). More details on the Nuzlocke Challenge can be found directly at this link: https://bulbapedia.bulbagarden.net/wiki/Nuzlocke_Challenge.$\underline{E}$ 
22. Nintendo of America, "Nintendo Corporate Mission

Statement," https://www.nintendo.com/corp/partnerships.jsp.

23. In the official Pokémon games, Pokémon can "faint" in battle if/when their health points (HP) reach zero. All official Pokémon games include a recovery system in which a player can take their fainted Pokémon to designated Pokémon Centers and completely revive them for a small fee (the fee is paid with fictional Poké dollars acquired during the normal course of gameplay and is not an additional out-of-game, redeemable purchase). This recovery system ensures that the player's Pokémon can never die. $\triangleq$

24. Bryan Bergerson, Developing Serious Games (Hingham, MA: Course Technology, 2006), 61-62.

25. Macdonald, "Why I'm a Full-Grown Adult Who Still Loves Pokémon."

26. Open-source can be defined as software for which the original source code is made freely available and may be modified or distributed to the general public. $\_$ 27. Hector Postigo, "Modding to the Big Leagues: Exploring the Space Between Modders and the Game Industry," First Monday 15, no. 5 (2010), http://firstmonday.org/ojs/index.php/fm/rt/printer Friendly/2972/2530. 28. This data collected was originally collected and analyzed by a Japanese media tracking company named Media Create. The reported information was found translated in English in an article written on December 1, 2014 by Ishaan, a contributor to the Siliconera.Com. Siliconera is a fan-made and operated gaming website. See Ishaan, "Pokémon's Audience Is Growing Older," Siliconera, December 1, 2014, http://www.siliconera.com/2014/12/01/pokemons-audience-growing-older/.. 29. David M. Sheridan, Jim Ridolfo, and Anthony J. Michel, Available Means of Persuasion: Mapping a Theory and Pedagogy of Multimodal Public Rhetoric (Anderson, SC: Parlor Press, 2012). $ヒ$ 30. The First Things First (FTF) Manifesto was created in 1963 and published in 1964 by Ken Garland and twenty other digital designers, photographers, and students as a response to societal pressure for more "humanist" designs. This original manifesto was later revisited and revised in 2000 to reflect a more philosophical outlook on the responsibilities trained specialists such as digital designers have in resisting the promotion of capitalist ideologies in their work as cultural producers. More 
information on the First Things First Manifesto can be directly found at this link: http://www.designishistory.com/1960/first-things-first/.. \pm

31. Sheridan, Ridolfo, and Michel, Available Means of Persuasion, xii. $\subseteq$

32. Sheridan, Ridolfo, and Michel, Available Means of Persuasion. $\bullet$

33. Ian Bogost, "The Rhetoric of Video Games" in Ecology of Games: Connecting

Youth, Games, and Learning, ed. Katie Salen (Cambridge, MA: MIT Press, 2008), 119.

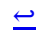

34. Poor, “Computer Game Modders," 1250.

35. Poor, “Computer Game Modders," 1253-1254.

36. Postigo, "Modding to the Big Leagues."

37. Sheridan, Ridolfo, and Michel, Available Means of Persuasion. $\subseteq$

38. Bogost, Persuasive Games; Bogost, "The Rhetoric of Video Games."

39. Walter Scacchi, "Computer Game Mods, Modders, Modding, and the Mod

Scene," First Monday 15, no. 5

(2010), http://firstmonday.org/ojs/index.php/fm/rt/printerFriendly/2965/2526.

40. Rhetorical velocity is a term used to describe how rhetoricians may strategically theorize and anticipate a third party recomposition of a text. For example, a blogger may anticipate the negative and positive ways their online audience may react to a certain kind of post, and how that post may be re-contextualized or edited to serve a rhetorical purpose outside of what the author originally intended. Ridolfo and DeVoss “Composing for Recomposition: Rhetorical Velocity and Delivery," Kairos: $A$ Journal of Rhetoric, Technology, and Pedagogy 13, no. 2 (2009). $ヒ$

41. Jens Seiffert and Howard Nothhaft, "The Missing Media: the Procedural Rhetoric of Computer Games," Public Relations Review 41 (2015):

255, http://dx.doi.org/10.1016/j.pubrev.2014.11.011.

42. Joshua Daniel-Wariya, “A Language of Play: New Media’s Possibility

Spaces," Computers and Composition 40 (2016):

33, http://dx.doi.org/10.1016/j.compcom.2016.03.011.

43. Bergeron, Developing Serious Games, xvii. $\_$ 
44. Poor, “Computer Games Modders,” 1264. 